\title{
PRICE SETTING DYNAMICS MODELING ON THE ONE PRODUCT MARKET UNDER UNCERTAINTY
}

\author{
Yevgeny Ye. Bizyanov \\ Donbass State Technical Institute, Alchevsk, Luhansk People's Republic \\ Nataliia A. Podgornaya \\ Donbass State Technical Institute, Alchevsk, Luhansk People's Republic
}

\begin{abstract}
This article discusses various options for studying the classic Evans model for assessing the dynamics of price setting for a new good in the conditions of uncertainty in the behavior of the market and the producer. The Evans model with fuzzy coefficients for supply and demand curves was used for modeling. As a result, it was found that when the level of opportunity for the parameters of the supply and demand curves change, the ranges of the equilibrium price will change, as well as the time of its establishment - the achievement of the equilibrium level. In addition, modeling was produced taking into account the lag in the supply from around the delay in the receipt of information on sales. The introduction of a fuzzy lag into the Evans model at a certain ratio of supply and demand parameters leads to significant price fluctuations, which can upset the equilibrium in the market, as well as lead to significant fluctuations in profit for the producer. The implementation of the Evans model with a lag in the proposal was carried out using the Simulink program of the Matlab. As a result of modeling, the dependences of the time for establishing the equilibrium price and the elevation of the price amplitude over its equilibrium value were obtained as a function of the opportunity's level.
\end{abstract}

Key words: Evans model, fuzzy set, fuzzy number, demand, supply, lag.

Citation. Bizyanov Ye.Ye., Podgornaya N.A. Price Setting Dynamics Modeling on the One Product Market Under Uncertainty. Vestnik Volgogradskogo gosudarstvennogo universiteta. Ekonomika [Journal of Volgograd State University. Economics], 2020, vol. 22, no. 4, pp. 41-49. (in Russian). DOI: https://doi.org/10.15688/ek.jvolsu.2020.4.4

\section{МОДЕЛИРОВАНИЕ ДИНАМИКИ УСТАНОВЛЕНИЯ ЦЕНЫ НА РЫНКЕ ОДНОГО ТОВАРА В УСЛОВИЯХ НЕОПРЕДЕЛЕННОСТИ}

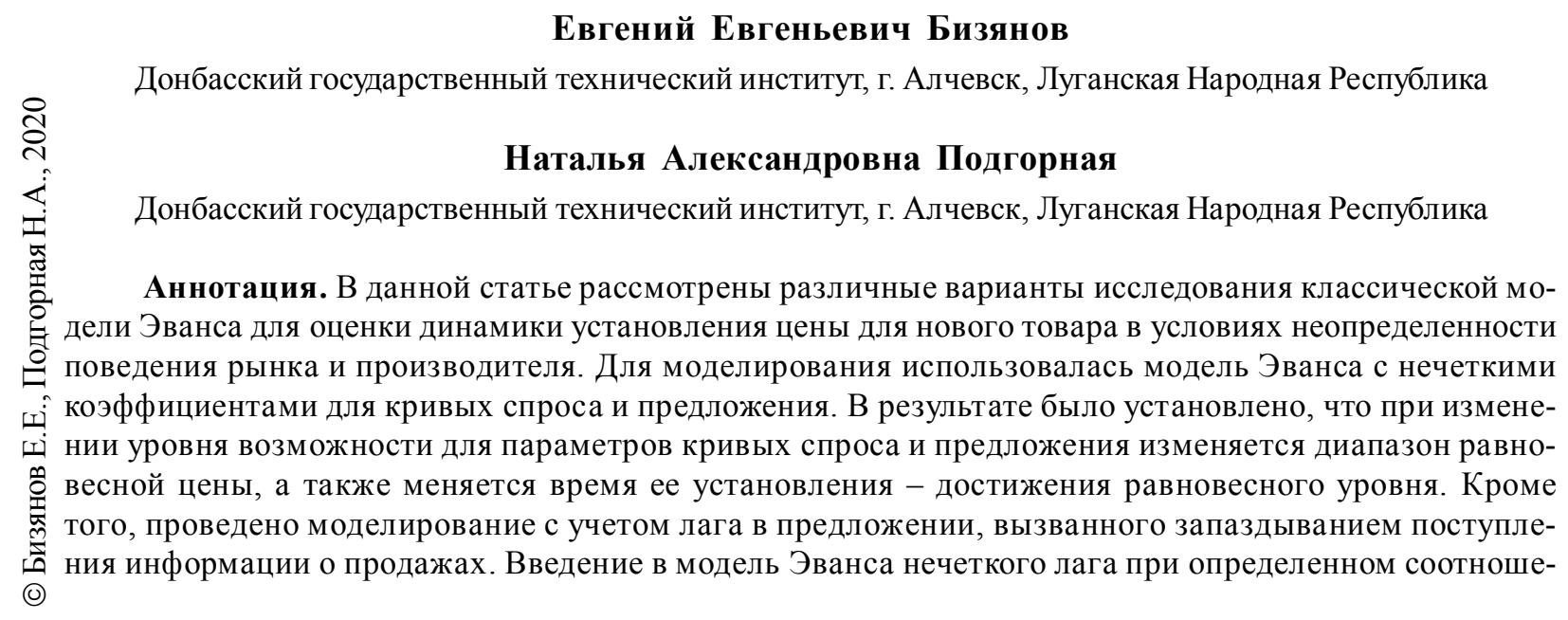


нии параметров спроса и предложения приводит к существенным колебаниям цены, что может нарушить равновесие на рынке, а также привести к существенным колебаниям прибыли для производителя. Реализация модели Эванса с лагом в предложении была проведена с использованием программы Simulink пакета Matlab. В результате моделирования получены зависимости времени установления равновесной цены и превышения амплитуды цены над ее равновесным значением, как функции от уровня возможности.

Ключевые слова: модель Эванса, нечеткое множество, нечеткое число, спрос, предложение, лаг.

Цитирование. Бизянов Е. Е., Подгорная Н. А. Моделирование динамики установления цены на рынке одного товара в условиях неопределенности // Вестник Волгоградского государственного университета. Экономика. - 2020. - Т. 22, № 4. - C. 41-49. - DOI: https://doi.org/10.15688/ek.jvolsu.2020.4.4

\section{Проблема и ее связь с научными и практическими задачами}

Модель Эванса позволяет получить равновесную цену и время ее установления при наличии параметров функций спроса и предложения [Колемаев, 2012, с. 197-198]. Как правило, коэффициенты уравнений спроса и предложения получают из статистических данных. Однако существует возможность возникновения ситуации, когда такая статистика отсутствует, либо необходимо оценить динамику установления цены для нового товара на рынке. В таких случаях возможным вариантом решения задачи может быть использование данных о спросе и предложении для товара-аналога или товара-субститута. Однако такой подход позволит получить единичное и не всегда точное значение установившейся цены, а также приблизительные параметры переходного процесса ее установления.

В классической модели Эванса реакция рынка (спрос) и производства (предложение) приняты мгновенными [Колемаев, 2012, c. 197]. В реальности же сведения о цене на рынке поступают с запаздыванием (лагом). Если лаг в спросе может отсутствовать или стремиться к нулю, то лаг в предложении всегда имеет место, так как вызван наличием времени на реализацию и обеспечение заказов оптовой и розничной торговли.

В статье [Пегат, 2011, с. 127-131] исследуется модель Эванса с учетом дискретного запаздывания скорости изменения цены товара. Запаздывание учитывается в предложении. Показано, что устойчивость достигается при определенных значениях цены и запаздывания.

В работе [Недосекин, 2003, с. 28-31] введение оператора дробного дифференцирова- ния позволило выяснить причины замедления динамики цены в момент времени, когда спрос и предложение равны.

Кусочно-постоянное запаздывание цены в предложении с введением функции подстройки цены предложения в модели Эванса позволило [Соколов и др., 2013, с. 33-36] сформулировать проблему в виде краевой задачи и найти условие существования решения уравнения с запаздыванием при различных значениях лага в цене.

В работах [Соколов и др., 2010; Шпилько и др., 2012] модель Эванса послужила основой для моделирования потребительского рынка, причем в [Соколов и др., 2010] введено запаздывание в процесс производства товара, а в [Шпилько и др., 2012] - в спрос на товар.

Следует отметить, что в [Колемаев, 2012; Пегат, 2011; Недосекин, 2003; Соколов и др., 2010; Соколов и др., 2013; Шпилько и др., 2012] анализируется модель Эванса с четкими значениями коэффициентов кривых спроса и предложения, что позволяет получить точечное решение. Для получения полной картины в условиях неопределенности при таком подходе необходимо выполнить множество расчетов (прогонов) модели, на основании которых можно будет принимать обоснованные решения.

Другой способ решения поставленной задачи - это использование теории нечетких множеств [Пшунетлев; Поддубный, 2006], позволяющей представить кривые спроса и предложения в виде некоторых множеств возможных значений.

Постановка задачи. Задачей моделирования является получение значений равновесной цены и времени ее установления на рынке одного товара в условиях неопределенности. 
E.E. Бизянов, Н.А. Подгорная. Моделирование динамики установления цены на рынке одного товара

\section{Изложение материала}

\section{и его результаты}

Уравнение модели Эванса имеет следующий вид [Колемаев, 2012, с. 197]:

$$
p^{\ni}(t)=\frac{d p}{d t}=\gamma(D(p)-S(p))
$$

где $\gamma$ - коэффициент пропорциональности; $D(p)-$ функции спроса, $S(p)$ - функции предложения.

При линейном представлении функций спроса $D(p)$ и предложения $S(p)$ их уравнения имеют вид [Колемаев, 2012, с. 197]:

$$
\begin{aligned}
& D(p)=a-b \cdot p, \\
& S(p)=\alpha-\beta \cdot p .
\end{aligned}
$$

Представим коэффициенты $a, b, \alpha, \beta$ в уравнениях нечеткими треугольными числами (рис. 1), которые можно интерпретировать как «примерно равно» [Пегат, 2011, с. 81]. Функция принадлежности нечеткого треугольного числа, приведенная на рисунке 1 , определяется тремя параметрами: модой $X_{C}$ и границами носителя $X_{R}$ и $X_{L}$.

Обычно уровень принадлежности $\mu_{\max }$ для $X=X_{C}$ равен единице. Значение переменной $X$ определяется так называемым уровнем возможности - $\alpha$-уровнем [Пегат, 2011, c. 39].

Часто нечеткие треугольные числа записывают в виде [Пегат, 2011, с. 29]:

$$
\widetilde{X}=\left(X_{L}, X_{C}, X_{R}\right) \text {. }
$$

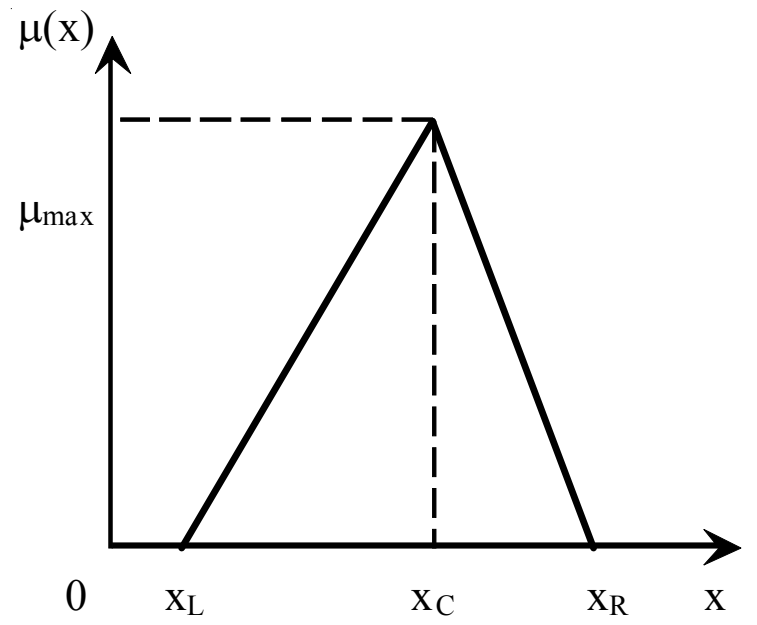

Рис. 1. Функция принадлежности нечеткого числа « $X$ примерно равен $X_{C}$ » Примечание. Составлено автором.

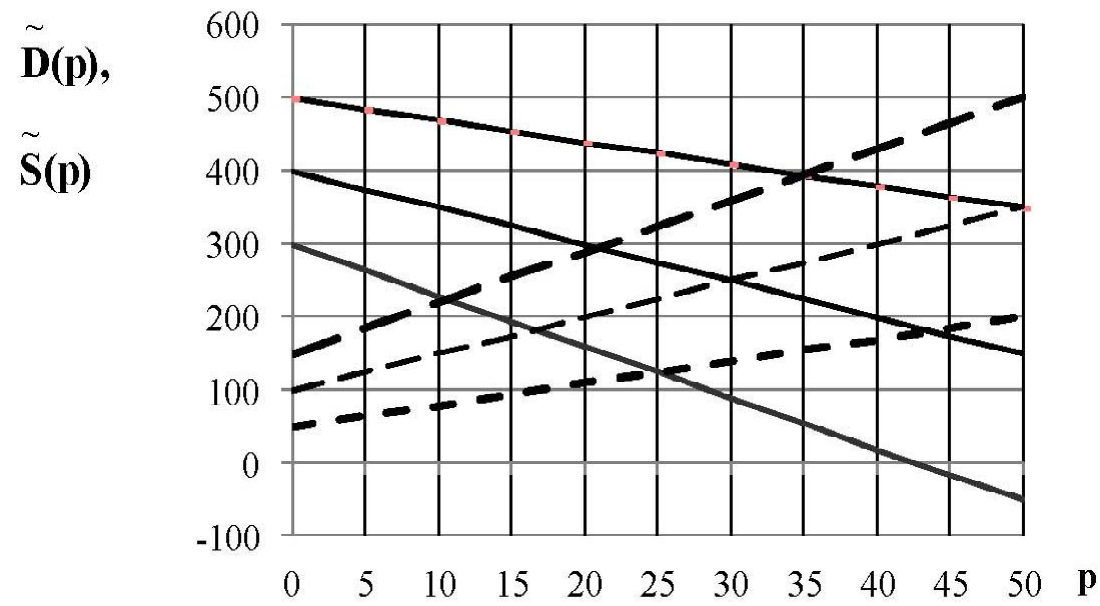

Рис. 2. Кривые спроса и предложения с нечеткими параметрами

Примечание. Составлено автором. 


\section{ЭКОНОМИЧЕСКАЯ ТЕОРИЯ}

Все последующие расчеты и моделирование будем производить, используя для коэффициентов уравнений спроса и предложения следующие значения:

$$
\begin{aligned}
& a=(300 ; 400 ; 500) ; \quad b=(3 ; 5 ; 7) ; \\
& \alpha=(50 ; 100 ; 150) ; \beta=(3 ; 5 ; 7) .
\end{aligned}
$$

Графики кривых спроса и предложения, построенные с использованием принятых нечетких значений параметров (4), приведены на рисунке 2.

Здесь средние по положению кривые соответствуют модам нечетких параметров, а нижние и верхние кривые отображают спрос и предложение для границ носителей параметров. При изменении $\alpha$-уровня с малым шагом от 0 до 1 таких кривых можно построить бесконечное множество.

Таким образом, вместо одной кривой спроса и одной кривой предложения мы получаем множество кривых, разброс параметров которых лежит в заданной области, а разнообразие значений указанных параметров позволяет получить множество сценариев динамики установления цены.
Для того чтобы разграничить параметры нечетких чисел и кривой предложения, $\alpha$-уровень для нечеткого числа будем обозначать как $A$.

Графики установления цены, построенные для уровней возможности $A=l$ (средняя кривая) и $A=0$ (верхняя кривая - при $a_{\max }$, $b_{\min }$ и $\alpha_{\min }, \beta_{\min }$; нижняя кривая - при $a_{\min }, b_{\max }$ и $\alpha_{\max }, \beta_{\max }$ ) показаны на рисунке 3 . Начальная цена принята равной $p(0)=5$.

Как следует из рисунка 3, при изменении уровня возможности А от 0 до 1 меняется как значение равновесной (установившейся) цены от 10 до 75, так и время ее установления меняется от 0,22 до 0,67 .

Теперь перейдем к рассмотрению модели Эванса с нечеткими коэффициентами кривых спроса и предложения и с лагом в предложении. Лаг также примем нечетким: $\tau=(0$; $0,5 ; 1)$.

На рисунке 4 показана реализация модели Эванса с лагом в предложении, реализованная в программе Simulink пакета Matlab [Дьяконов, 2005]. Здесь блоки-константы задают значения коэффициентов а и $\alpha$ для кривых спроса и предложения, $p(0)$ - начальную

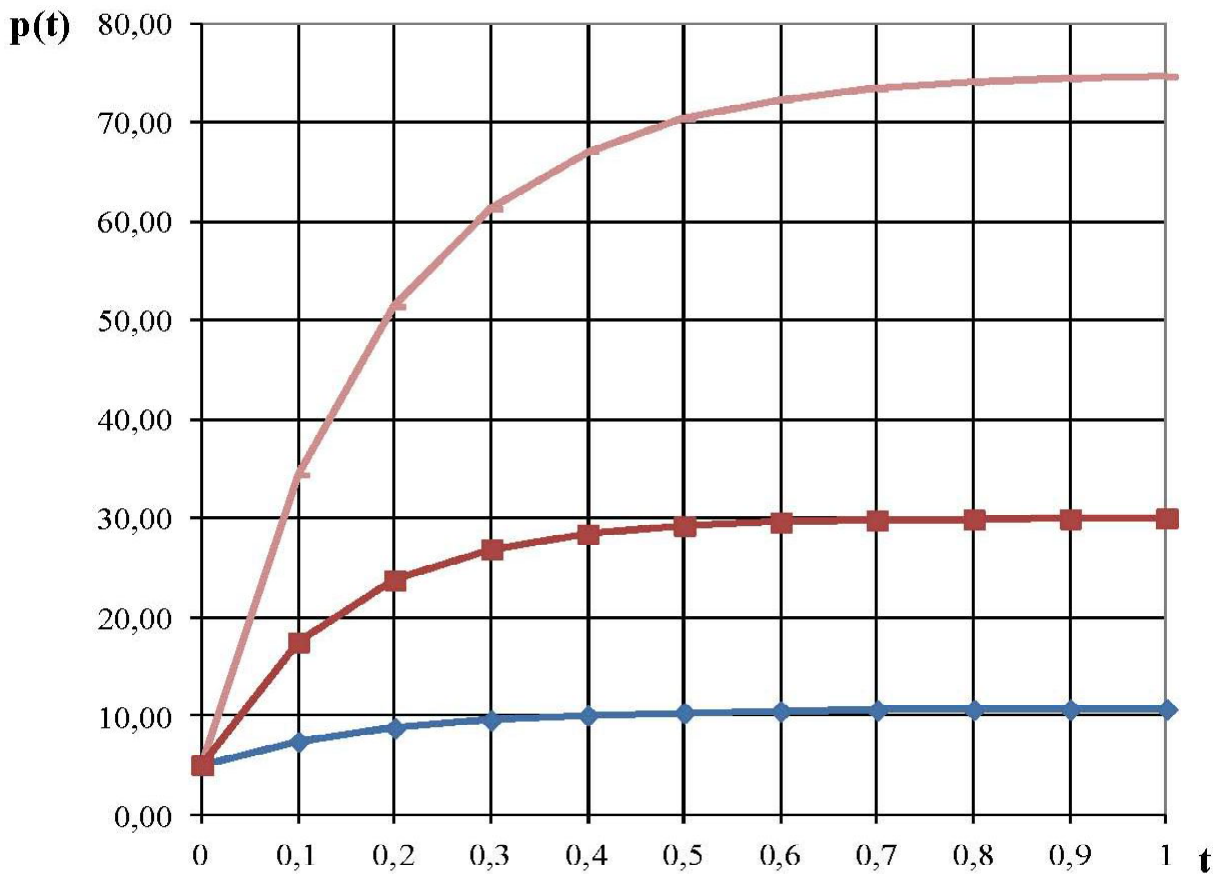

Рис. 3. Графики установления цены на рынке одного товара при различных уровнях возможности

Примечание. Составлено автором. 
E.E. Бизянов, Н.А. Подгорная. Моделирование динамики установления цены на рынке одного товара

цену, блоки-усилители - значения коэффициентов $b$ и $\beta$ для кривых спроса и предложения.

В модели, представленной на рисунке 4, лаг реализован в блоке чистого запаздывания Transport Delay. Решение дифференциального уравнения (1) производится с помощью блока интегрирования Integrator.

Результаты моделирования выводятся в блоки Scope: $p$ - цена, $W$ - выручка. Выручка рассчитывается в блоке Product как произведение текущей цены на величину спроса. Таблица меток времени и соответствующих им значений цены выводится в рабочее пространство Matlab с помощью блока To Workspace.
Результаты моделирования, полученные автором, приведены в таблице и на рисунке 5. В таблице равновесная цена обозначена как $p^{*}$, время установления цены - как $t_{y c m}$, а превышение амплитуды текущей цены над ее равновесным (установившимся) значением как $\sigma$. Графики на рисунке 5 обозначены цифрами, соответствующими вариантам расчета таблицы. Время установления цены определялось по правилу, принятому в теории регулирования [Бесекерский, 2003, с. 209]: переходный процесс считается завершенным, если наблюдаемая величина вошла в 5 \%-й «коридор», построенный относительно установившегося значения, и больше не вышла из него.

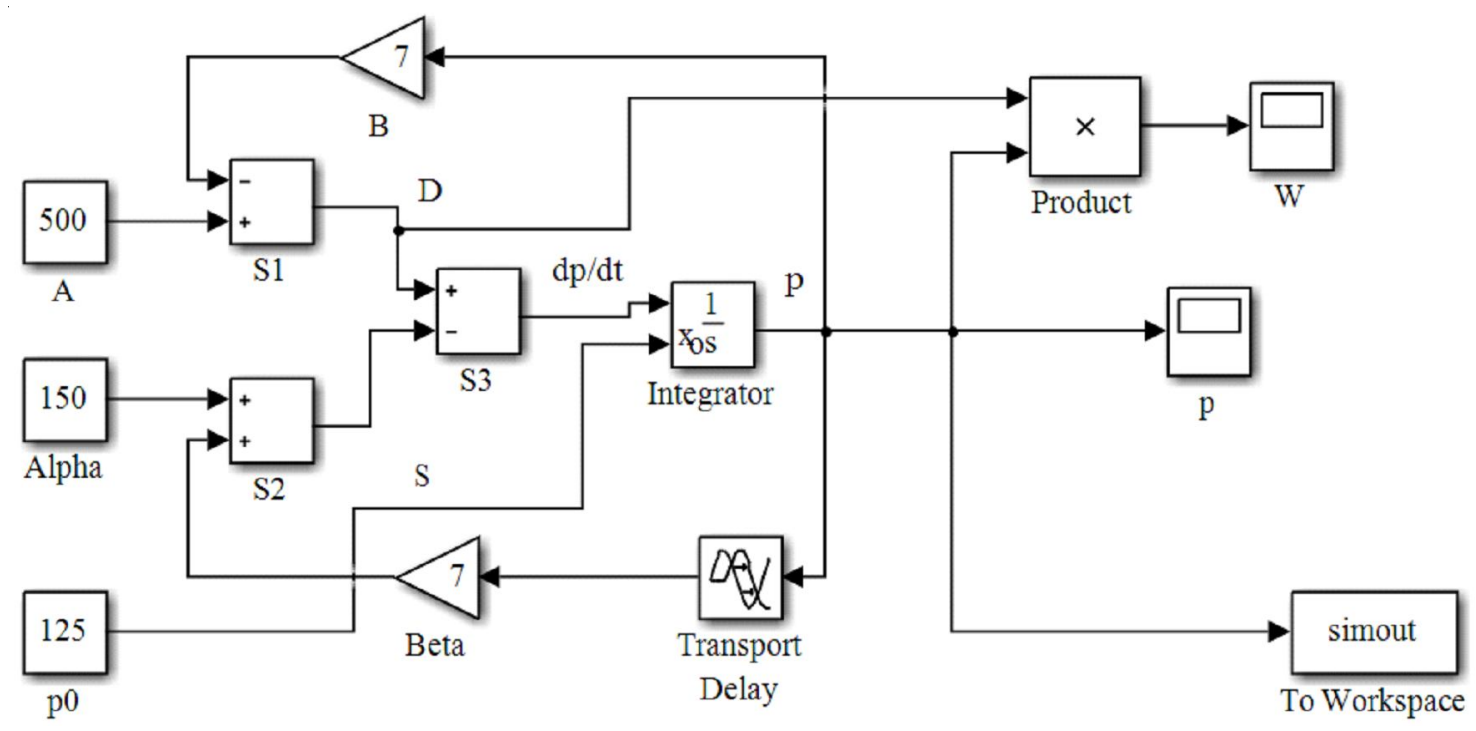

Рис. 4. Модель Эванса с лагом - реализация в Simulink

Примечание. Составлено автором.

Результаты моделирования

\begin{tabular}{|c|c|c|c|c|c|}
\hline \multirow{2}{*}{ Параметр } & \multicolumn{5}{|c|}{ Вариант } \\
\cline { 2 - 6 } & 1 & 2 & 3 & 4 & 5 \\
\hline$\mu$ & 0 & 0 & 0,5 & 0,5 & 1 \\
\hline$a$ & 300 & 500 & 350 & 450 & 400 \\
\hline$b$ & 3 & 7 & 4 & 6 & 5 \\
\hline$\alpha$ & 50 & 150 & 75 & 125 & 100 \\
\hline$\beta$ & 3 & 7 & 4 & 6 & 5 \\
\hline$\tau$ & 0 & 1 & 0,25 & 0,75 & 0,5 \\
\hline$p^{*}$ & 41,6 & 25 & 34,3 & 27,1 & 30 \\
\hline$t_{y c m}$ & 0,45 & 36 & 1,5 & 13 & 3,5 \\
\hline$\sigma, \%$ & 0 & 79,7 & 31,9 & 78,9 & 65,2 \\
\hline
\end{tabular}

Примечание. Составлено автором. 


\section{ЭКОНОМИЧЕСКАЯ ТЕОРИЯ}

На рисунке 6 показаны области изменения времени установления цены $t_{y c m}$ и превышения амплитуды текущей цены над ее равновесным значением $\sigma$ как функций от уровня возможности $\mu$.
На рисунке 7 приведен график динамики изменения выручки от времени в нечеткой модели Эванса для варианта 2 таблицы (соответствует уровню возможности $\mu=0)$. Как можно видеть из полученной зависимости,

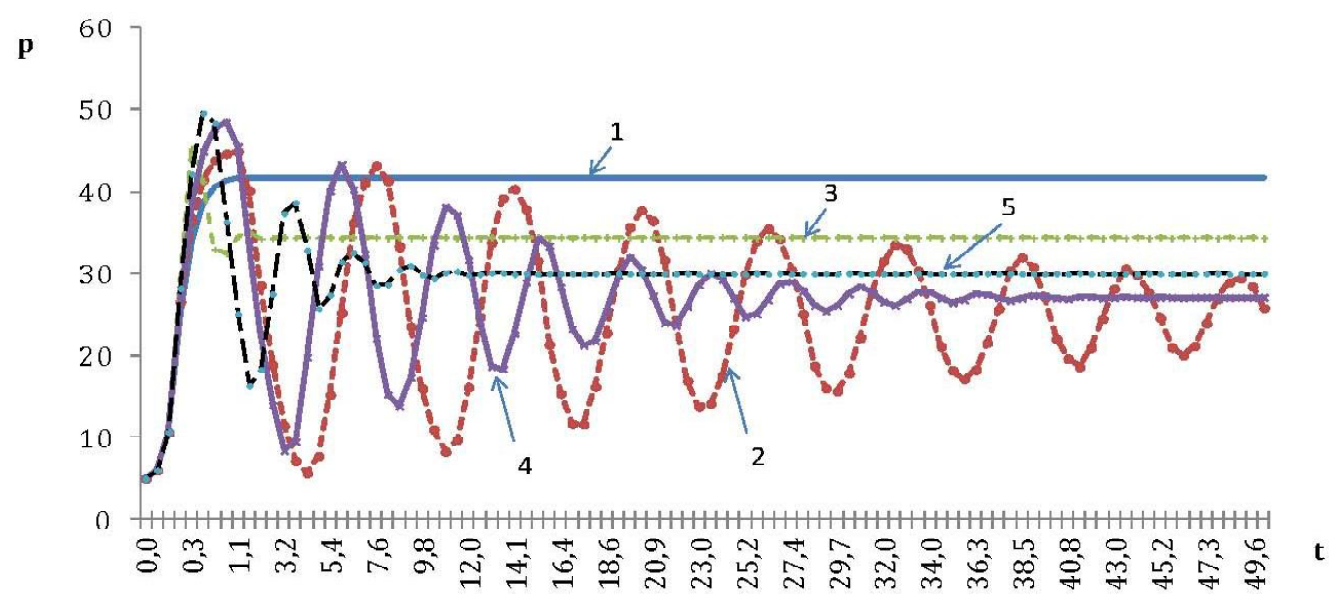

Рис. 5. Графики динамики изменения цены от времени в нечеткой модели Эванса при различных уровнях возможности

Примечание. Составлено автором.

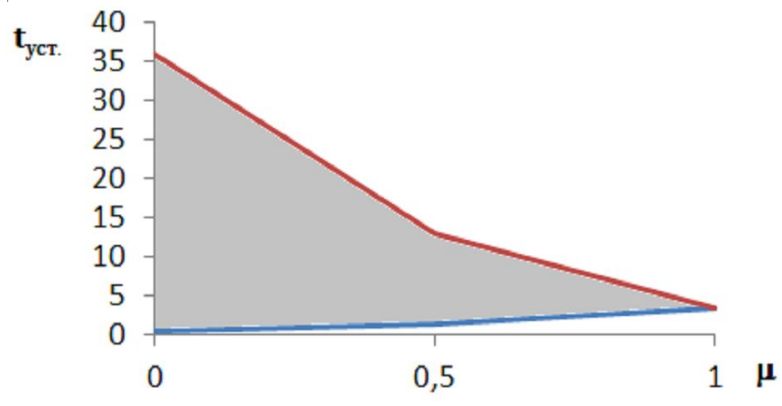

a

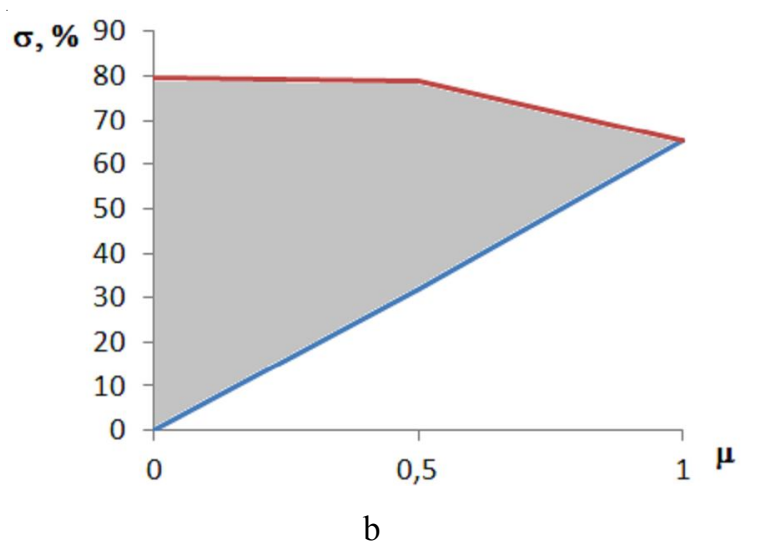

Рис. 6. Зависимости времени установления цены (а) и превышения амплитуды цены (б) от уровня возможности в нечеткой модели Эванса с лагом

Примечание. Составлено автором. 


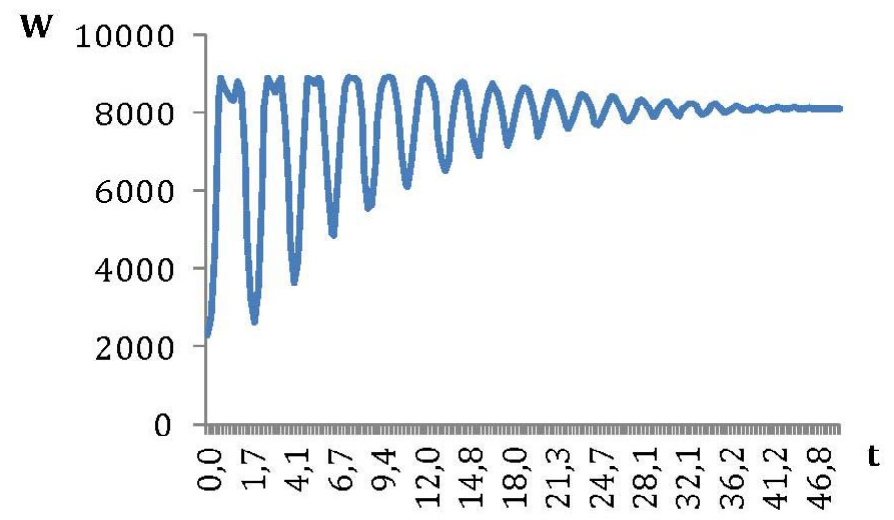

Рис. 7. График динамики изменения выручки от времени в нечеткой модели Эванса для варианта 2 таблицы 1

Примечание. Составлено автором.

наличие лага приводит к существенным колебаниям выручки.

Данное явление может привести не только к нарушению баланса на рынке, но и проблемам с управлением оборотными средствами у производителя, что в конечном счете приведет к дестабилизации производственного процесса.

\section{Выводы и направление дальнейших исследований}

Проведенное моделирование установления цены на рынке одного товара с помощью модели Эванса с нечеткими коэффициентами показало, что при изменении уровня возможности для параметров спроса и предложения изменяется равновесная цена и время ее установления. Введение в модель Эванса нечеткого лага при определенном соотношении параметров спроса и предложения приводит к существенным колебаниям цены, что может нарушить равновесие на рынке, а также вызвать проблемы у производителя, что в конечном счете приведет к дестабилизации производственного процесса.

В дальнейших исследованиях необходимо произвести учет ограничений предельных величин спроса (объем рынка не бесконечен) и предложения (возможности производства также не бесконечны), а также исследовать случаи, когда уровень возможности для спроса, предложения и величины лага будет различным.

\section{СПИСОК ЛИТЕРАТУРЫ}

Бесекерский, В. А. Теория систем автоматического регулирования / В. А. Бесекерский, Е. П. Попов. - 4-е изд., перераб. и доп. СПб. : Профессия, 2003. - 752 с.

Дьяконов, В. П. МATLAB R2006/2007/2008 + Simulink 5/6/7: основы применения / В. П. Дьяконов. 2-е изд., перераб. и доп. - М. : СОЛОН-Пресс, 2005. - 799 с. : ил., табл. ; 22 см. - (Серия «Библиотека профессионала»).

Колемаев, В. А. Математическая экономика : учебник для студентов высших учебных заведений, обучающихся по экономическим специальностям / В. А. Колемаев. - 3-е стер. изд. - М. : ЮНИТИ, 2012. - 399 с. : ил.

Недосекин, А. О. Методологические основы моделирования финансовой деятельности с использованием нечетко-множественных описаний : дис. ... д-ра экон. наук : 08.00.13 / А. О. Недосекин ; СПбГУЭФ. - СПб., 2003. - 280 с.

Пегат, А. Нечеткое моделирование и управление / А. Пегат ; пер. с англ. - М. : БИНОМ ; Лаборатория знаний, 2011.-798 с. : ил. -(Серия «Адаптивные и интеллектуальные системы»).

Поддубный, В. В. Исследование динамической модели рынка вальрасовского типа со многими товарами / В. В. Поддубный, Е. А. Сухарева // Вестник Томского государственного университета. - 2006. - № 293. - С. 53-58.

Пшунетлев, А. А. Имитационное моделирование регионального потребительского рынка / А. А. Пшунетлев // Научный журнал КубГАУ. -2014. - № 98 (04). - Электрон. текстовые дан. - Режим доступа: http://ej.kubagro.ru/ 2014/04/pdf/38.pdf. - Загл. с экрана. 
Соколов, В. А. Исследование устойчивости линейной модели Вальраса-Эванса-Самуэльсона с учетом зависимости спроса и предложения от скорости изменения цены / В. А. Соколов, А. Ю. Фомина // Вестник Пермского государственного технического университета. 2010. - № 15. - С. 28-31.

Соколов, В. А. Об одной краевой задаче в модели Вальраса-Эванса-Самуэльсона рынка одного товара / В. А. Соколов, Н. А. Стрикун / Перспективы науки. - 2013. - № 8 (47). - С. 127-131.

Шпилько, Я. Е. Параметризация уравнения Самуэльсона в модели Эванса об установлении равновесной цены на рынке одного товара / Я. Е. Шпилько, А. А. Соломко, Р. И. Паровик // Вестник КРАУНЦ. Физико-математические науки. - 2012. - № 2 (5). - С. 33-36.

\section{REFERENCES}

Besekerskij V.A. Teorija sistem avtomaticheskogo regulirovanija [Theory of Automatic Control Systems]. Saint Petersburg, Professiya Publ., 2003. 752 p.

D'jakonov V.P. MATLAB R2006/2007/2008 + Simulink 5/6/7: osnovy primenenija [MATLAB R2006 / 2007/2008 + Simulink 5/6/7: Application Basics]. Moscow, SOLON-Press, 2005. 799 p.: fig., tab.; $22 \mathrm{~cm}$. (Seriya «Biblioteka professionala» [Series "Library of the Professional"]).

Kolemaev V.A. Matematicheskaja jekonomika : uchebnik dlja studentov vysshih uchebnyh zavedenij, obuchajushhihsja pojekonomicheskim special'nostjam [Mathematical Economics: A Textbook for Students of Higher Educational Institutions Studying in Economic Specialties]. Moscow, UNITI Publ., 2012. 399 p.: fig.

Nedosekin A.O. Metodologicheskie osnovy modelirovanija finansovoj dejatel'nosti s ispol'zovaniem nechetko-mnozhestvennyh opisanij: diss. ... d-ra.ekon. nauk [Methodological Foundations for Modeling Financial Activities
Using Fuzzy-Multiple Descriptions. Dr. econ. sci. diss.]. Saint Petersburg, SPbGUEF, 2003. 280 p.

Pegat A. Nechetkoe modelirovanie i upravlenie [Fuzzy Modeling and Control]. Moscow, BINOM Publ., Laboratoriya znaniy Publ., 2011. 798 p.: fig. (Seriya "Adaptivnye i intellektualnye sistemy» [Series "Adaptive and Intelligent Systems"]).

Poddubnyj V.V. Issledovanie dinamicheskoj modeli rynka val'rasovskogo tipa so mnogimi tovarami [Research of a Dynamic Model of the Walras Type Market with Many Goods]. Vestnik Tomskogo gosudarstvennogo universiteta [Bulletin of the Tomsk State University], 2006, no. 293, pp. 53-58.

Pshunetlev A.A. Imitacionnoe modelirovanie regional'nogo potrebitel'skogo rynka [Imitation Modeling of the Regional Consumer Market]. Nauchnyy zhurnal KubGAU [Scientific Journal of KubSAU], 2014, no. 98 (04). URL: http:// ej.kubagro.ru/2014/04/pdf/38.pdf.

Sokolov V.A. Issledovanie ustojchivosti linejnoj modeli Val'rasa-Jevansa-Samujel'sona s uchetom zavisimosti sprosa i predlozhenija ot skorosti izmenenija ceny [Investigation of the Stability of the Linear Walras-Evans-Samuelson Model Taking into Account the Dependence of Supply and Demand on the Rate of Price Change]. Vestnik Permskogo gosudarstvennogo tekhnicheskogo universiteta [Bulletin of the Perm State Technical University], 2010, no. 15, pp. 28-31.

Sokolov V.A. Ob odnoj kraevoj zadache v modeli Val'rasa-Jevansa-Samujel'sona rynka odnogo tovara [On one boundary value problem in the Walras-Evans-Samuelson model of the market for one product]. Perspektivy nauki [Perspectives of Science], 2013, no. 8 (47), pp. 127-131.

Shpil'ko Ja.E. Parametrizacija uravnenija Samujel'sona v modeli Jevansa ob ustanovlenii ravnovesnoj ceny na rynke odnogo tovara [Parametrization of the Samuelson equation in the Evans model of establishing the equilibrium price in the market for one product]. Vestnik KRAUNTs. Fizikomatematicheskie nauki [Bulletin of KRAUNTS Phys.-Math. Science], 2012, no. 2 (5), pp. 33-36. 


\section{Information About the Authors}

Yevgeny Ye. Bizyanov, Doctor of Sciences (Economics), Professor, Department of Specialized Computer Systems, Donbass State Technical Institute, Prosp. Lenina, 16, 94204 Alchevsk, Luhansk People's Republic, bpeelecs@gmail.com, https://orcid.org/0000-0003-2841-3619

Nataliia A. Podgornaya, Candidate of Sciences (Engineering), Senior Lecturer, Department of Higher Mathematics, Donbass State Technical Institute, Prosp. Lenina, 16, 94204 Alchevsk, Luhansk People’s Republic, nataly.podgorna@gmail.com, https://orcid.org/0000-0002-2337-8108

\section{Информация об авторах}

Евгений Евгеньевич Бизянов, доктор экономических наук, профессор кафедры специализированных компьютерных систем, Донбасский государственный технический институт, просп. Ленина, 16, 94204 г. Алчевск, Луганская Народная Республика, bpeelecs@gmail.com, https://orcid.org/0000-0003-2841-3619

Наталья Александровна Подгорная, кандидат технических наук, старший преподаватель кафедры высшей математики, Донбасский государственный технический институт, просп. Ленина, 16, 94204 г. Алчевск, Луганская Народная Республика, nataly.podgorna@gmail.com, https://orcid.org/0000-0002-2337-8108 